\title{
A Class of Absolutely Continuous BIVARIATE DistRIBUtions
}

\author{
Debasis Kundu ${ }^{\dagger} \&$ Rameshwar D. Gupta ${ }^{\ddagger}$
}

\begin{abstract}
Block and Basu bivariate exponential distribution is one of the most popular absolutely continuous bivariate distribution. Extensive work has been done on the Block and Basu bivariate exponential model over the past several decades. Interestingly it is observed that the Block and Basu bivariate exponential model can be extended to the Weibull model also. We call this new model as the Block and Basu bivariate Weibull model. We consider different properties of the Block and Basu bivariate Weibull model. The Block and Basu bivariate Weibull model has four unknown parameters and the maximum likelihood estimators cannot be obtained in closed form. To compute the the maximum likelihood estimators directly, one needs to solve a four dimensional optimization problem. We propose to use the EM algorithm for computing the maximum likelihood estimators of the unknown parameters. The proposed EM algorithm can be carried out by solving one non-linear equation at each EM step. Our method can be used to compute the maximum likelihood estimators for the Block and Basu bivariate exponential model also. One data analysis has been preformed for illustrative purpose.
\end{abstract}

KEYwORDs: Block and Basu bivariate exponential model; Joint probability density function; Conditional probability density function; Maximum likelihood estimators; Asymptotic Distribution; Fisher information matrix; EM algorithm.

$\dagger$ Department of Mathematics and Statistics, Indian Institute of Technology Kanpur, Kanpur, Pin 208016,

INDIA. Phone no. 91-512-2597141, e-mail: kundu@iitk.ac.in. Corresponding author.

$\dagger$ Department of Computer Science and Statistics, The University of New Brunswick at Saint John, New Brunswick, Canada E2L 4L5. Part of the work has been supported by a discovery grant from NSERC, CANADA. 


\section{INTRODUCTION}

Block and Basu [3] obtained the bivariate exponential distribution (BBBE) from the MarshallOlkin bivariate exponential (MOBE) distribution by removing the singular part and retaining only the absolutely continuous part. Although MOBE is a singular bivariate exponential distribution, BBBE distribution enjoys all the properties of an absolutely continuous distribution. Because of this reason, BBBE is a very popular bivariate distributions. It has been used extensively for data analysis purposes, even though it is known that the marginals of BBBE are not exponential unlike MOBE model.

Along the same line as MOBE distribution, see for example Marshall and Olkin [11], Block and Basu bivariate Weibull (BBBW) distribution has been defined. BBBW model has been obtained from the Marshall-Olkin bivariate Weibull (MOBW) model by removing the singular part and that makes BBBW distribution as an absolutely continuous bivariate distribution. Clearly, it is a more flexible model than BBBE model because of the presence of the shape parameter. Although extensive work has been done on BBBE model, not that much of attention has been paid on BBBW model. The reason might be; in spite of the fact $\mathrm{BBBW}$ is more flexible than BBBE, computationally it may not be very tractable. In fact computing the maximum likelihood estimators (MLEs) of the unknown parameters of MMBW model is not a trivial issue.

In this paper we provide different properties of BBBW model. We discuss different computational issues associated in computing the parameters of BBBW model. First we consider the computation of MLEs of the four unknown parameters of BBBW model. It is observed that MLEs cannot be obtained in explicit form as expected, and they can be obtained by solving a multidimensional optimization problem. It is observed that the EM algorithm can be used quite effectively to compute MLEs of BBBW parameters. At each step (iteration), 
one needs to solve only a one dimensional optimization problem, and we have proposed a simple procedure to solve this problem. We have also provided the observed and expected Fisher information matrix. The expected Fisher information matrix provides the dispersion matrix of the asymptotic distribution of MLEs and the observed Fisher information matrix is needed to compute the approximate confidence intervals of the unknown parameters.

Although MOBE or BBBE model has been proposed quite some times back, only recently Karlis [7] proposed an efficient estimation technique to compute the unknown parameters of MOBE model. Moreover, in spite of the fact BBBE model has been derived from the Marshall-Olkin bivariate exponential model, it is not clear how the method of Karlis [7] can

be used to compute MLEs of BBBE model. Note that BBBE model can be obtained as a special case of BBBW model and the proposed EM algorithm can be used very easily for BBBE model also. In fact in case of BBBE model, at each EM step no non-linear equation needs to be solved.

Rest of the paper is organized as follows. In section 2 we introduce the model and provide different properties of the model in section 3. The maximum likelihood estimators are discussed in section 4. Analysis of one data set has been presented in section 5 and finally we conclude the paper in section 6 .

\section{BBBW: Model Description}

We use the following notation for the rest of the paper. If $X$ has a univariate Weibull distribution with the shape and scale parameters as $\alpha>0$ and $\lambda>0$ respectively, then for $x>0$, the probability density function (PDF) is defined as follows;

$$
f_{W E}(x ; \alpha, \lambda)=\alpha \lambda x^{\alpha-1} e^{-\lambda x^{\alpha}}
$$


The corresponding survival function (SF) and the hazard function (HF) will be denoted by $S_{W E}(x ; \alpha, \lambda)$ and $h_{W E}(x ; \alpha, \lambda)$ respectively. A Weibull distribution with the shape parameter $\alpha$ and the scale parameter $\lambda$ will be denoted by $\operatorname{WE}(\alpha, \lambda)$.

Suppose $U_{0}$ follows $(\sim) \mathrm{WE}\left(\alpha, \lambda_{0}\right), U_{1} \sim \mathrm{WE}\left(\alpha, \lambda_{1}\right)$ and $U_{2} \sim \mathrm{WE}\left(\alpha, \lambda_{2}\right)$ and they are mutually independent. If $X_{1}=\min \left\{U_{0}, U_{1}\right\}$ and $X_{2}=\min \left\{U_{0}, U_{2}\right\}$, then the joint distribution function of $\left(X_{1}, X_{2}\right)$ is called the Marshall-Olkin bivariate Weibull distribution. The joint survival function of $\left(X_{1}, X_{2}\right)$ can be written for $z=\max \left\{x_{1}, x_{2}\right\}$ as;

$$
\begin{aligned}
S_{M O}\left(x_{1}, x_{2}\right)= & P\left(X_{1}>x_{1}, X_{2}>x_{2}\right)=P\left(U_{0}>z, U_{1}>x_{1}, U_{2}>x_{2}\right) \\
= & S_{W E}\left(x_{1} ; \alpha, \lambda_{1}\right) S_{W E}\left(x_{2} ; \alpha, \lambda_{2}\right) S_{W E}\left(z ; \alpha, \lambda_{0}\right) \\
& =\left\{\begin{array}{ccc}
S_{W E}\left(x_{1} ; \alpha, \lambda_{1}\right) S_{W E}\left(x_{2} ; \alpha, \lambda_{0}+\lambda_{2}\right) & \text { if } & 0<x_{1}<x_{2}<\infty \\
S_{W E}\left(x_{1} ; \alpha, \lambda_{0}+\lambda_{1}\right) S_{W E}\left(x_{2} ; \alpha, \lambda_{2}\right) & \text { if } & 0<x_{2}<x_{1}<\infty \\
S_{W E}\left(x ; \alpha, \lambda_{0}+\lambda_{1}+\lambda_{2}\right) & \text { if } & 0<x_{1}=x_{2}=x<\infty .
\end{array}\right.
\end{aligned}
$$

It may be observed that the joint survival function of $\left(X_{1}, X_{2}\right)$ can be written as a mixture of an absolutely continuous part and a singular part as follows;

$$
S_{M O}\left(x_{1}, x_{2}\right)=\frac{\lambda_{1}+\lambda_{2}}{\lambda_{0}+\lambda_{1}+\lambda_{2}} S_{a}\left(x_{1}, x_{2}\right)+\frac{\lambda_{0}}{\lambda_{0}+\lambda_{1}+\lambda_{2}} S_{s}\left(x_{1}, x_{2}\right),
$$

where $S_{a}(\cdot, \cdot)$ is the absolutely continuous part and $S_{s}(\cdot, \cdot)$ is the singular part. Note that for $z=\max \left\{x_{1}, x_{2}\right\}$,

$$
S_{s}\left(x_{1}, x_{2}\right)=S_{W E}\left(z ; \alpha, \lambda_{0}+\lambda_{1}+\lambda_{1}\right)
$$

and $S_{a}\left(x_{1}, x_{2}\right)$ can be obtained by subtraction as

$$
S_{a}\left(x_{1}, x_{2}\right)=\frac{\lambda_{0}+\lambda_{1}+\lambda_{2}}{\lambda_{1}+\lambda_{2}} e^{-\lambda_{1} x_{1}^{\alpha}} e^{-\lambda_{2} x_{2}^{\alpha}} e^{-\lambda_{0} z^{\alpha}}-\frac{\lambda_{0}}{\lambda_{1}+\lambda_{2}} e^{-\left(\lambda_{0}+\lambda_{1}+\lambda_{2}\right) z^{\alpha}} .
$$

Note that BBBW distribution can be obtained from MOBW distribution by removing the singular part and keeping only the continuous part. The joint PDF of BBBW can be 
written as

$$
f_{B B}\left(y_{1}, y_{2}\right)=\left\{\begin{array}{lll}
c f_{1}\left(y_{1}, y_{2}\right)=c f_{W E}\left(y_{1} ; \alpha, \lambda_{1}\right) f_{W E}\left(y_{2} ; \alpha, \lambda_{0}+\lambda_{2}\right) & \text { if } \quad 0<y_{1}<y_{2} \\
c f_{2}\left(y_{1}, y_{2}\right)=c f_{W E}\left(y_{1} ; \alpha, \lambda_{0}+\lambda_{1}\right) f_{W E}\left(y_{2} ; \alpha, \lambda_{2}\right) & \text { if } \quad 0<y_{2}<y_{1},
\end{array}\right.
$$

here $c$ is the normalizing constant and $c=\frac{\lambda_{0}+\lambda_{1}+\lambda_{2}}{\lambda_{1}+\lambda_{2}}$. Therefore, the joint PDF of $\left(Y_{1}, Y_{2}\right)$ can be written as (5) and it will be denoted by $\operatorname{BBBW}\left(\alpha, \lambda_{0}, \lambda_{1}, \lambda_{2}\right)$. The joint survival function of $Y_{1}$ and $Y_{2}$ is $S_{a}(\cdot, \cdot)$. The joint $\mathrm{PDF}$ of $\left(Y_{1}, Y_{2}\right)$ is unimodal and the surface plot of $f_{B B}\left(y_{1}, y_{2}\right)$ for different values of $\alpha$ and $\lambda_{0}$, keeping $\lambda_{1}=\lambda_{2}=1$, are provided in Figure 1. From the Figure 1, it is clear that it can take different shapes, which can be very useful for data analysis purposes.

Figure 1(a)

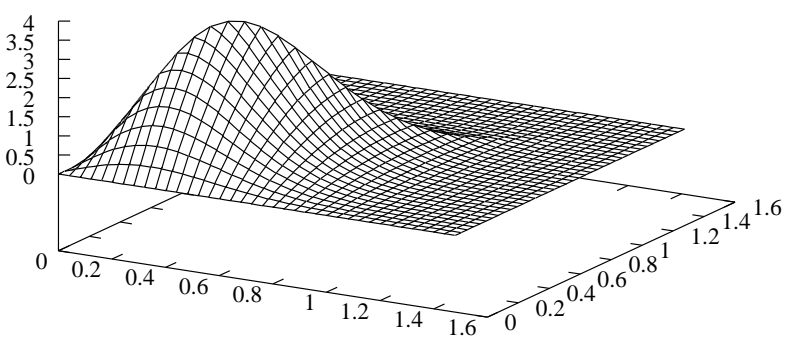

Figure 1(c)

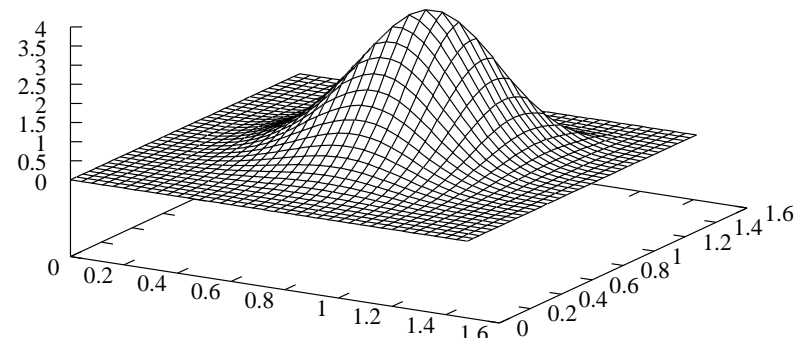

Figure 1(b)

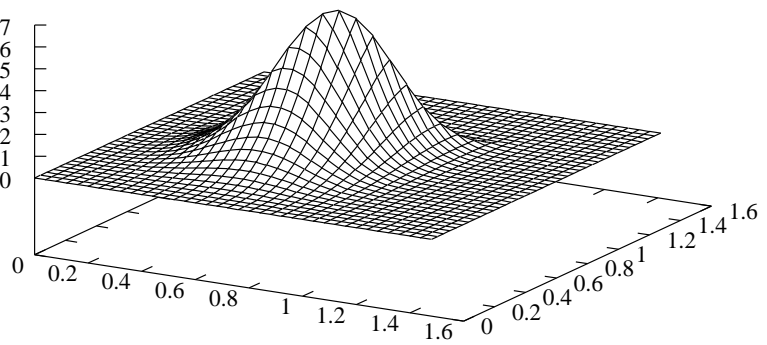

Figure 1(d)

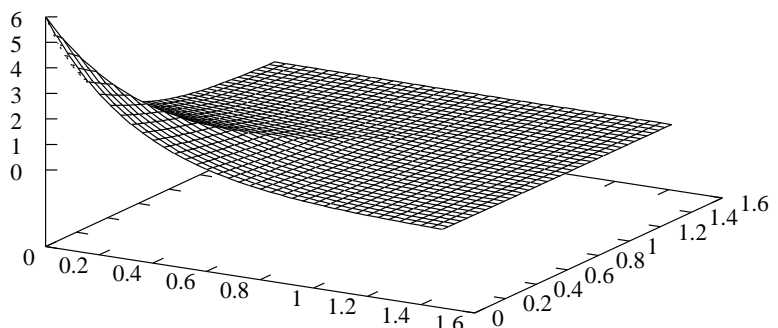

Figure 1: The joint PDFs of $\left(Y_{1}, Y_{2}\right)$. (a) $\alpha=3, \lambda_{0}=4.0$, (b) $\alpha=4, \lambda_{0}=4.0$, (c) $\alpha=4$, $\lambda_{0}=1.0$, (d) $\alpha=1, \lambda_{0}=2.0$,

From the joint $\operatorname{PDF} f_{B B}\left(y_{1}, y_{2}\right)$, it is immediate that when $\lambda_{0}=0$, then $Y_{1}$ and $Y_{2}$ become independent and both of them have Weibull distributions. For $\lambda_{0}>0, Y_{1}$ and $Y_{2}$ are dependent. It will be proved later that for $\lambda_{0}>0, Y_{1}$ and $Y_{2}$ are positively correlated. 
From the construction of the Block and Basu bivariate Weibull distribution, it is immediate that if $\left(X_{1}, X_{2}\right) \sim \operatorname{MOBW}\left(\alpha, \lambda_{0}, \lambda_{1}, \lambda_{2}\right)$, and $\left(Y_{1}, Y_{2}\right)=\left(X_{1}, X_{2}\right)$, given that $X_{1}, \neq X_{2}$, then $\left(Y_{1}, Y_{2}\right) \sim \operatorname{BBBW}\left(\alpha, \lambda_{0}, \lambda_{1}, \lambda_{2}\right)$. Therefore, the generation from the BBBW is very simple. We can adopt the following simple generation technique to generate random samples from BBBW.

Algorithm to Generate From BBBW

- Step 1: Generate $U_{0} \sim \mathrm{WE}\left(\alpha, \lambda_{0}\right), U_{1} \sim \mathrm{WE}\left(\alpha, \lambda_{1}\right)$ and $U_{2} \sim \mathrm{WE}\left(\alpha, \lambda_{2}\right)$ independently.

- Step 2: If $U_{0}<U_{1}$ and $U_{0}<U_{2}$ go back to Step 1 otherwise set $Y_{1}=\min \left\{U_{0}, U_{1}\right\}$ and $Y_{2}=\min \left\{U_{0}, U_{2}\right\}$.

\section{Different Properties}

In this section we provide different basic properties of the BBBW model. First we provide the marginal and conditional distributions of BBBW model.

Theorem 3.1 If $\left(Y_{1}, Y_{2}\right) \sim \operatorname{BBBW}\left(\alpha, \lambda_{0}, \lambda_{1}, \lambda_{2}\right)$, then the marginal PDFs of $Y_{1}$ and $Y_{2}$ are

$$
f_{Y_{1}}\left(y_{1}\right)=c f_{W E}\left(y_{1} ; \alpha, \lambda_{0}+\lambda_{1}\right)-c \frac{\lambda_{0}}{\lambda_{0}+\lambda_{1}+\lambda_{2}} f_{W E}\left(y_{1} ; \alpha, \lambda_{0}+\lambda_{1}+\lambda_{2}\right)
$$

and

$$
f_{Y_{2}}\left(y_{2}\right)=c f_{W E}\left(y_{2} ; \alpha, \lambda_{0}+\lambda_{2}\right)-c \frac{\lambda_{0}}{\lambda_{0}+\lambda_{1}+\lambda_{2}} f_{W E}\left(y_{2} ; \alpha, \lambda_{0}+\lambda_{1}+\lambda_{2}\right)
$$

respectively, where $c$ is the same as before.

PROOF: They can be obtained by routine calculations.

Now we will discuss some of the basic properties of the marginals of BBBW. We mainly mention the properties of $Y_{1}$. The properties of $Y_{2}$ are exactly the same. From Theorem 3.1, 
it is clear as expected that the distribution of $Y_{1}$ is not Weibull distribution in general. For $\lambda_{0}=0$ or $\lambda_{2}=0$, the distribution of $Y_{1}$ becomes Weibull. Although the PDF of $Y_{1}$ is not Weibull in general, but the shape of the PDF of $Y_{1}$ is very similar to the PDF of a Weibull distribution. The hazard function of $Y_{1}$ is

$$
h_{Y_{1}}(y)=\left(\lambda_{0}+\lambda_{1}+\lambda_{2}\right) \alpha y^{\alpha-1}\left[1-\frac{\lambda_{2}}{\lambda_{0}+\lambda_{1}+\lambda_{2}-\lambda_{0} e^{-\lambda_{2} y^{\alpha}}}\right] \text {. }
$$

The hazard function (HF) of $Y_{1}$ can be increasing, decreasing or constant depending on the values of $\alpha$ and $\lambda$ 's. The PDFs and HFs of $Y_{1}$ for different values of $\alpha$ and $\lambda_{0}$ for $\lambda_{1}=$ $\lambda_{2}=1$ are provided in Figure 2. The PDF of $Y_{1}$ can be written as a weighted version of
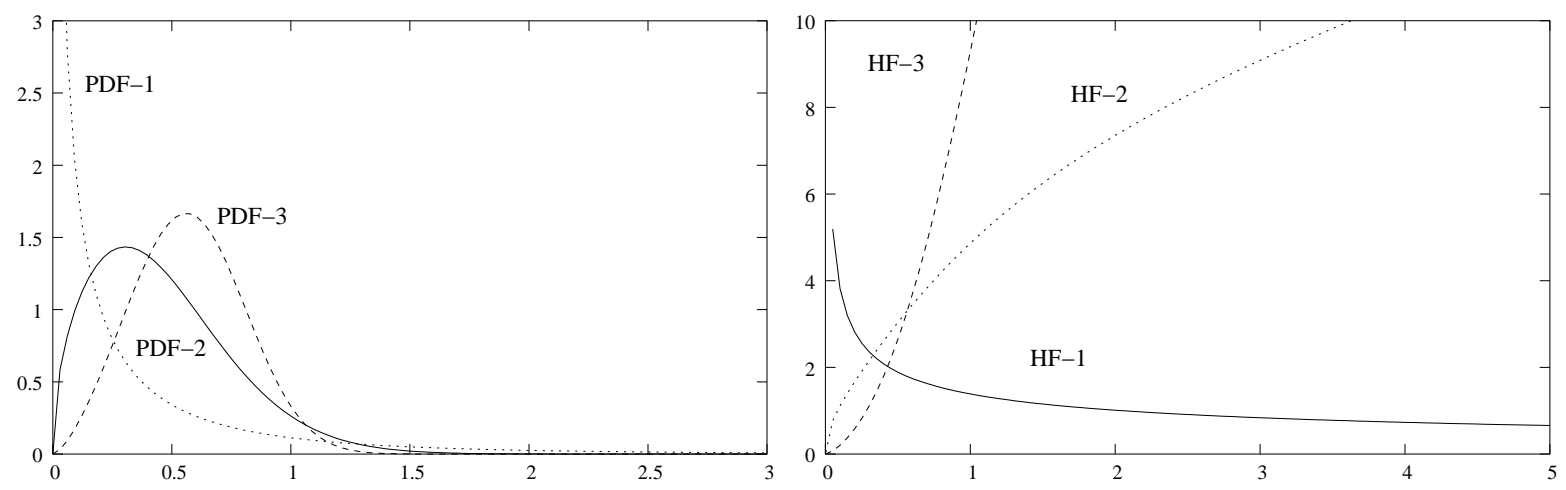

Figure 2: The PDFs and HFs of $Y_{1}$ for, PDF-1/HF-1: $\alpha=0.5, \lambda_{0}=2.0, \mathrm{PDF}-2 / \mathrm{HF}-2: \alpha=$ 1.5, $\lambda_{0}=2.5$, PDF-3/HF-3: $\alpha=2.5, \lambda_{0}=3.0$.

$\mathrm{WE}\left(\alpha, \lambda_{0}+\lambda_{1}\right)$ distribution with the weight function proportional to

$$
w_{1}(y)=\lambda_{0}+\lambda_{1}-\lambda_{0} e^{-\lambda_{2} y^{\alpha}}
$$

Therefore many properties which are available for the weighted distributions can be applied here also. Moreover, all the moments of $Y_{1}$ exist and they can be written in terms of gamma functions. Now we will discuss the conditional PDFs.

Theorem 3.2: If $\left(Y_{1}, Y_{2}\right) \sim \operatorname{BBBW}\left(\alpha, \lambda_{0}, \lambda_{1}, \lambda_{2}\right)$, then the conditional PDFs of $Y_{1} \mid Y_{2}=y_{2}$ 
and $Y_{2} \mid Y_{1}=y_{1}$ are

$$
f_{Y_{1} \mid Y_{2}=y_{2}}\left(y_{1}\right)=\left\{\begin{array}{ccc}
\frac{\lambda_{0}+\lambda_{2}}{\lambda_{2}+\lambda_{0}\left(1-e^{-\lambda_{1} y_{2}^{\alpha}}\right)} f_{W E}\left(y_{1} ; \alpha, \lambda_{1}\right) & \text { if } \quad y_{1}<y_{2} \\
\frac{\lambda_{2}}{e^{-\lambda_{0} y_{2}^{\alpha}}\left(\lambda_{2}+\lambda_{0}\left(1-e^{-\lambda_{1} y_{2}^{\alpha}}\right)\right)} f_{W E}\left(y_{1} ; \alpha, \lambda_{0}+\lambda_{1}\right) & \text { if } \quad y_{2}<y_{1}
\end{array}\right.
$$

and

$$
f_{Y_{2} \mid Y_{1}=y_{1}}\left(y_{2}\right)=\left\{\begin{array}{cl}
\frac{\lambda_{1}}{e^{-\lambda_{0} y_{1}^{\alpha}}\left(\lambda_{1}+\lambda_{0}\left(1-e^{-\lambda_{2} y_{1}^{\alpha}}\right)\right)} f_{W E}\left(y_{2} ; \alpha, \lambda_{0}+\lambda_{2}\right) & \text { if } \quad y_{1}<y_{2} \\
\frac{\lambda_{0}+\lambda_{1}}{\lambda_{1}+\lambda_{0}\left(1-e^{-\lambda_{2} y_{1}^{\alpha}}\right)} f_{W E}\left(y_{2} ; \alpha, \lambda_{2}\right) & \text { if } \quad y_{2}<y_{1}
\end{array}\right.
$$

respectively.

PRoOF: They can be obtained by routine calculation.

Theorem 3.1 and Theorem 3.2 also can be used to generate samples from BBBW. The bivariate survival function of $Y_{1}$ and $Y_{2}$ is

$$
S_{B B}\left(y_{1}, y_{2}\right)= \begin{cases}c e^{-\lambda_{1} y_{1}^{\alpha}} e^{-\left(\lambda_{0}+\lambda_{2}\right) y_{2}^{\alpha}}-\frac{\lambda_{0}}{\lambda_{1}+\lambda_{2}} e^{-\left(\lambda_{0}+\lambda_{1}+\lambda_{2}\right) y_{2}^{\alpha}} & \text { if } \quad y_{1}<y_{2} \\ c e^{-\left(\lambda_{0}+\lambda_{1}\right) y_{1}^{\alpha}} e^{-\lambda_{2} y_{2}^{\alpha}}-\frac{\lambda_{0}}{\lambda_{1}+\lambda_{2}} e^{-\left(\lambda_{0}+\lambda_{1}+\lambda_{2}\right) y_{1}^{\alpha}} & \text { if } \quad y_{2}<y_{1}\end{cases}
$$

and the marginal survival functions of $Y_{1}$ and $Y_{2}$ are

$$
\begin{aligned}
& S_{Y_{1}}\left(y_{1}\right)=c e^{-\left(\lambda_{0}+\lambda_{1}\right) y_{1}^{\alpha}}-\frac{\lambda_{0}}{\lambda_{1}+\lambda_{2}} e^{-\left(\lambda_{0}+\lambda_{1}+\lambda_{2}\right) y_{1}^{\alpha}} \quad \text { and } \\
& S_{Y_{2}}\left(y_{2}\right)=c e^{-\left(\lambda_{0}+\lambda_{2}\right) y_{2}^{\alpha}}-\frac{\lambda_{0}}{\lambda_{1}+\lambda_{2}} e^{-\left(\lambda_{0}+\lambda_{1}+\lambda_{2}\right) y_{2}^{\alpha}},
\end{aligned}
$$

respectively.

The bivariate failure rate as defined by Basu [2] can be written as

$$
r\left(y_{1}, y_{2}\right)=\frac{f_{B B}\left(y_{1}, y_{2}\right)}{S_{B B}\left(y_{1}, y_{2}\right)}=\left\{\begin{array}{llc}
\frac{h_{W E}\left(y_{1} ; \alpha, \lambda_{1}\right) h_{W E}\left(y_{2} ; \alpha, \lambda_{0}+\lambda_{2}\right)}{1-\frac{\lambda_{0}}{\lambda_{0}+\lambda_{1}+\lambda_{2}} e^{-\lambda_{1}\left(y_{2}^{\alpha}-y_{1}^{\alpha}\right)}} & \text { if } & y_{1}<y_{2} \\
\frac{h_{W E}\left(y_{1} ; \alpha, \lambda_{0}+\lambda_{1}\right) h_{W E}\left(y_{2} ; \alpha, \lambda_{2}\right)}{1-\frac{\lambda_{0}}{\lambda_{0}+\lambda_{1}+\lambda_{2}} e^{-\lambda_{1}\left(y_{1}^{\alpha}-y_{2}^{\alpha}\right)}} & \text { if } & y_{1}>y_{2}
\end{array}\right.
$$

It is clear that when $\alpha=1$, it reduces to Block and Basu bivariate failure rate. When they are independent (14) reduces to product of two Weibull hazards as expected. Moreover, interestingly, along the curve $y_{1}^{\alpha}=y_{2}^{\alpha}+b$, for some constant $b, r\left(y_{1}, y_{2}\right)$ is an increasing, decreasing or constant according as $\alpha>1, \alpha<1$ or $\alpha=1$ respectively. 
The hazard gradients, see Johnson and Kotz [5], of BBBW are

$$
h_{1}\left(y_{1}, y_{2}\right)=-\frac{\partial}{\partial y_{1}} \ln S\left(y_{1}, y_{2}\right)=\left\{\begin{array}{ccc}
\frac{h_{W E}\left(y_{1} ; \alpha, \lambda_{1}\right)}{1-\frac{\lambda_{0}}{\lambda_{0}+\lambda_{1}+\lambda_{2}} e^{-\lambda_{1}\left(y_{2}^{\alpha}-y_{1}^{\alpha}\right)}} & \text { if } & y_{1}<y_{2} \\
\frac{h_{W E}\left(y_{1} ; \alpha, \lambda_{0}+\lambda_{1}\right)}{1-\frac{\lambda_{0}}{\lambda_{0}+\lambda_{1}+\lambda_{2}} e^{-\lambda_{2}\left(y_{1}^{\alpha}-y_{2}^{\alpha}\right)}}+\frac{h_{W E}\left(y_{1} ; \alpha, \lambda_{0}+\lambda_{1}+\lambda_{2}\right)}{1-\frac{\lambda_{0}+\lambda_{1}+\lambda_{2}}{\lambda_{0}} e^{-\lambda_{2}\left(y_{2}^{\alpha}-y_{1}^{\alpha}\right)}} & \text { if } & y_{2}<y_{1}
\end{array}\right.
$$

and

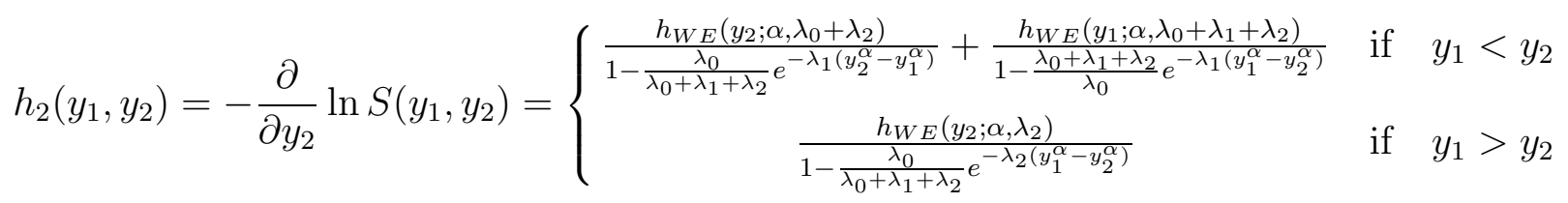

Now we provide the total positivity result of $Y_{1}$ and $Y_{2}$, for identical marginals.

Theorem 3.3: If $\left(Y_{1}, Y_{2}\right) \sim \operatorname{BBBW}\left(\alpha, \lambda_{0}, \lambda, \lambda\right)$, then $\left(Y_{1}, Y_{2}\right)$ has the total positivity of order two $\left(\mathrm{TP}_{2}\right)$ property.

Proof: Note that $\left(Y_{1}, Y_{2}\right)$ has the $\mathrm{TP}_{2}$ property if and only if for any $y_{11}, y_{12}, y_{21}, y_{22}$, whenever, $0<y_{11}<y_{12}$ and $0<y_{21}<y_{22}$, then

$$
f_{Y_{1}, Y_{2}}\left(y_{11}, y_{21}\right) f_{Y_{1}, Y_{2}}\left(y_{12}, y_{22}\right) \geq f_{Y_{1}, Y_{2}}\left(y_{12}, y_{21}\right) f_{Y_{1}, Y_{2}}\left(y_{11}, y_{22}\right) .
$$

Now we consider different cases as follows;

CASE 1: $y_{21}<y_{22}<y_{11}<y_{12}$.

In this case

$$
f_{Y_{1}, Y_{2}}\left(y_{11}, y_{21}\right) f_{Y_{1}, Y_{2}}\left(y_{12}, y_{22}\right)-f_{Y_{1}, Y_{2}}\left(y_{12}, y_{21}\right) f_{Y_{1}, Y_{2}}\left(y_{11}, y_{22}\right)=0
$$

CASE 2: $y_{21}<y_{11}<y_{22}<y_{12}$

In this case it can be easily observed by simple calculation that, to prove (15) is equivalent to prove

$$
e^{-\lambda_{0} y_{11}^{\alpha}} \geq e^{-\lambda_{0} y_{22}^{\alpha}}
$$

Clearly (16) is true as $y_{11}<y_{22}$. Similarly, for other cases also it can be proved along the similar line. 
Since $\left(Y_{1}, Y_{2}\right)$ have $\mathrm{TP}_{2}$ property, therefore, for any non-decreasing function $U(\cdot, \cdot)$ and $V(\cdot, \cdot), \operatorname{Cov}\left(U\left(Y_{1}, Y_{2}\right), V\left(Y_{1}, Y_{2}\right)\right)>0$. It implies that $Y_{1}$ and $Y_{2}$ are also positively correlated. $\left(Y_{1}, Y_{2}\right)$ will also be positively likelihood ratio dependent, i.e. it satiafies (15), see Lehmann [9]. Moreover, since the joint density function is $\mathrm{TP}_{2}$, the joint survival function is also $\mathrm{TP}_{2}$, which is equivalent to right corner set increasing, i.e. $P\left(Y_{1}>y_{1}, Y_{2}>y_{2} \mid Y_{1}>y_{1}^{\prime}, Y_{2}>y_{2}^{\prime}\right)$ is nondecreasing in $y_{1}^{\prime}, y_{2}^{\prime}$, for every choice of $y_{1}, y_{2}$, see Harris [4].

The following results will be useful for data analysis purposes. The proofs are quite trivial and therefore omitted.

Theorem 3.4: Suppose $\left(Y_{1}, Y_{2}\right) \sim \operatorname{BBBW}\left(\alpha, \lambda_{0}, \lambda_{1}, \lambda_{2}\right)$.

(a) The stress-strength parameter $R=P\left(Y_{1}<Y_{2}\right)$, has the following form;

$$
R=\frac{\lambda_{1}}{\lambda_{1}+\lambda_{2}}
$$

(b) $\min \left\{Y_{1}, Y_{2}\right\} \sim \operatorname{WE}\left(\alpha, \lambda_{0}+\lambda_{1}+\lambda_{2}\right)$.

(c) $Y_{1} \mid\left\{Y_{1}<Y_{2}\right\} \sim \mathrm{WE}\left(\alpha, \lambda_{0}+\lambda_{1}+\lambda_{2}\right)$

(d) $Y_{2} \mid\left\{Y_{2}<Y_{1}\right\} \sim \mathrm{WE}\left(\alpha, \lambda_{0}+\lambda_{1}+\lambda_{2}\right)$.

The BBBW model satisfies all the regularity conditions for the MLEs to be consistent and asymptotically normal and we can state the following result:

THeOREm 3.5: If $\widehat{\alpha}, \widehat{\lambda}_{0}, \widehat{\lambda}_{1}$ and $\widehat{\lambda}_{2}$ are the MLEs of $\alpha, \lambda_{0}, \lambda_{1}$ and $\lambda_{2}$ respectively, then

$$
\sqrt{n}\left\{\widehat{\alpha}-\alpha, \widehat{\lambda}_{0}-\lambda_{0}, \widehat{\lambda}_{1}-\lambda_{1}, \widehat{\lambda}_{2}-\lambda_{2}\right\} \rightarrow N_{4}\left(0, I^{-1}\right)
$$

here $I$ is the Fisher information matrix and the exact expression of $I$ is provided in the Appendix B.

In the next section we provide the method of finding the MLEs. 


\section{Maximum Likelihood Estimators:}

In this section we are mainly discussing about the computation of the MLEs of the unknown parameters $\alpha, \lambda_{0}, \lambda_{1}$ and $\lambda_{2}$ of the BBBW distribution, when we have a random sample $\left\{\left(y_{11}, y_{21}\right), \ldots,\left(y_{1 n}, y_{2 n}\right)\right\}$. We use the following notation: $I_{1}=\left\{i: y_{1 i}<y_{2 i}\right\}$ and $I_{2}=\{i$ :

$\left.y_{1 i}>y_{2 i}\right\},\left|I_{1}\right|=n_{1}$ and $\left|I_{2}\right|=n_{2}$. Based on the above notation, the log-likelihood function can be written as

$$
\begin{aligned}
l\left(\alpha, \lambda_{0}, \lambda_{1}, \lambda_{2}\right)= & n \ln \left(\lambda_{0}+\lambda_{1}+\lambda_{2}\right)-n \ln \left(\lambda_{1}+\lambda_{2}\right)+\sum_{i \in I_{1}} \ln f_{W E}\left(y_{1 i} ; \alpha, \lambda_{1}\right) \\
& +\sum_{i \in I_{1}} \ln f_{W E}\left(y_{2 i} ; \alpha, \lambda_{0}+\lambda_{2}\right)+\sum_{i \in I_{2}} \ln f_{W E}\left(y_{1 i} ; \alpha, \lambda_{0}+\lambda_{1}\right) \\
& +\sum_{i \in I_{2}} \ln f_{W E}\left(y_{1 i} ; \alpha, \lambda_{2}\right) \\
= & n \ln \left(\lambda_{0}+\lambda_{1}+\lambda_{2}\right)-n \ln \left(\lambda_{1}+\lambda_{2}\right)+(\alpha-1) \sum_{I}\left[\ln y_{1 i}+\ln y_{2 i}\right]+2 n \ln \alpha \\
& +n_{1}\left(\ln \lambda_{1}+\ln \left(\lambda_{0}+\lambda_{2}\right)\right)+n_{2}\left(\ln \lambda_{2}+\ln \left(\lambda_{0}+\lambda_{1}\right)\right)-\lambda_{1} \sum_{i \in I} y_{1 i}^{\alpha}-\lambda_{2} \sum_{i \in I} y_{2 i}^{\alpha} \\
& -\lambda_{0}\left(\sum_{i \in I_{1}} y_{1 i}^{\alpha}+\sum_{i \in I_{2}} y_{2 i}^{\alpha}\right)
\end{aligned}
$$

Clearly, the MLEs of $\alpha, \lambda_{0}, \lambda_{1}$ and $\lambda_{2}$ cannot be obtained in explicit forms. They can be obtained only by solving four equations in four unknowns. We treat this problem as a missing value problem. Observe that if all the $U_{0}, U_{1}$ and $U_{2}$ are known, the maximum likelihood estimates of the unknown parameters can be obtained by solving a one dimensional optimization problem. We try to exploit this property to treat this problem as a missing value problem through EM algorithm.

First let us look at the complete observations and then we provide the missing observations. Let us define a pair of random variables $\left(\Delta_{1}, \Delta_{2}\right)$ associated with each $\left(X_{1}, X_{2}\right)$ as 
follows;

$$
\left(\Delta_{1}, \Delta_{2}\right)=\left\{\begin{array}{lll}
(0,0) & \text { if } & X_{1}=U_{0}, X_{2}=U_{0} \\
(0,2) & \text { if } & X_{1}=U_{0}, X_{2}=U_{2} \\
(1,0) & \text { if } & X_{1}=U_{1}, X_{2}=U_{0} \\
(1,2) & \text { if } & X_{1}=U_{1}, X_{2}=U_{2} .
\end{array}\right.
$$

If a sample is obtained from $\left(X_{1}, X_{2}, \Delta_{1}, \Delta_{2}\right)$, we call it as the complete observation. Note that if we have a random sample of size $m$ from $\left(X_{1}, X_{2}, \Delta_{1}, \Delta_{2}\right)$, then the MLEs of the unknown parameters can be obtained as a one dimensional optimization problem as follows. Suppose we have the following observations; $\left\{\left(x_{1 i}, x_{2 i}, \delta_{1 i}, \delta_{2 i}\right), i=1, \ldots m\right\}$. We use the following notation;

$$
\begin{aligned}
I_{0} & =\left\{i ; x_{1 i}=x_{2 i}=x_{i}, \delta_{1 i}=\delta_{2 i}=0\right\}, \quad I_{02}=\left\{i ; x_{1 i}>x_{2 i}, \delta_{1 i}=0, \delta_{2 i}=2\right\} \\
I_{10} & =\left\{i ; x_{1 i}<x_{2 i}, \delta_{1 i}=1, \delta_{2 i}=0\right\}, \quad I_{121}=\left\{i ; x_{1 i}<x_{2 i}, \delta_{1 i}=1, \delta_{2 i}=2\right\} \\
I_{122} & =\left\{i ; x_{1 i}>x_{2 i}, \delta_{1 i}=1, \delta_{2 i}=2\right\} .
\end{aligned}
$$

The likelihood contributions of the observations from the set $I_{0}, I_{02}, I_{10}, I_{121}, I_{122}$ are $\alpha \lambda_{0} x_{i}^{\alpha-1} e^{-\left(\lambda_{0}+\lambda_{1}+\lambda_{2}\right) x_{i}^{\alpha}}, \quad \alpha \lambda_{2} x_{2 i}^{\alpha-1} e^{-\lambda_{2} x_{2 i}^{\alpha}} \quad \alpha \lambda_{0} x_{1 i}^{\alpha-1} e^{-\left(\lambda_{0}+\lambda_{1}\right) x_{1 i}^{\alpha}}, \quad \alpha \lambda_{1} x_{1 i}^{\alpha-1} e^{-\lambda_{1} x_{1 i}^{\alpha}}$ $\alpha \lambda_{0} x_{2 i}^{\alpha-1} e^{-\left(\lambda_{0}+\lambda_{2}\right) x_{2 i}^{\alpha}}, \alpha \lambda_{1} x_{1 i}^{\alpha-1} e^{-\lambda_{1} x_{1 i}^{\alpha}} \alpha \lambda_{2} x_{2 i}^{\alpha-1} e^{-\left(\lambda_{0}+\lambda_{2}\right) x_{2 i}^{\alpha}}, \alpha \lambda_{1} x_{1 i}^{\alpha-1} e^{-\left(\lambda_{0}+\lambda_{1}\right) x_{1 i}^{\alpha}} \alpha \lambda_{2} x_{2 i}^{\alpha-1} e^{-\lambda_{2} x_{2 i}^{\alpha}}$ respectively. It is clear from the above likelihood contributions, that given the complete observations, the MLEs of the unknown parameters can be obtained by solving one nonlinear equation only.

In our case the missing observations are the whole set $I_{0}$. We do not observe $I_{10}$ and $I_{121}$ separately, but we observe $I_{1}=I_{10} \cup I_{121}$. Therefore, in $I_{1}, \delta_{1 i}$ is known, but $\delta_{2 i}$ is missing. Similarly, we do not observe $I_{02}$ and $I_{122}$ separately, we observe $I_{2}=I_{02} \cup I_{122}$. In this case $\delta_{1 i}$ is missing but $\delta_{2 i}$ is known. The observations in Table 1 will be useful for constructing E-step of the EM algorithm. 
Table 1: All possible cases of $U_{0}, U_{1}, U_{2}$, corresponding probabilities and $\left(\Delta_{1}, \Delta_{2}\right)$

\begin{tabular}{|c|c|c|c|c|}
\hline Different Cases & Probability & $\left(\Delta_{1}, \Delta_{2}\right)$ & $X_{1} \& X_{2}$ & Set \\
\hline$U_{0}<U_{1}<U_{2}$ & $\frac{\lambda_{0} \lambda_{1}}{\left(\lambda_{1}+\lambda_{2}\right)\left(\lambda_{0}+\lambda_{1}+\lambda_{2}\right)}$ & $(0,0)$ & $X_{1}=X_{2}$ & $I_{0}$ \\
\hline$U_{0}<U_{2}<U_{1}$ & $\frac{\lambda_{0} \lambda_{2}}{\left(\lambda_{1}+\lambda_{2}\right)\left(\lambda_{0}+\lambda_{1}+\lambda_{2}\right)}$ & $(0,0)$ & $X_{1}=X_{2}$ & $I_{0}$ \\
\hline$U_{1}<U_{0}<U_{2}$ & $\frac{\lambda_{0} \lambda_{1}}{\left(\lambda_{0}+\lambda_{2}\right)\left(\lambda_{0}+\lambda_{1}+\lambda_{2}\right)}$ & $(1,0)$ & $X_{1}<X_{2}$ & $I_{10}$ \\
\hline$U_{1}<U_{2}<U_{0}$ & $\frac{\lambda_{1} \lambda_{2}}{\left(\lambda_{0}+\lambda_{2}\right)\left(\lambda_{0}+\lambda_{1}+\lambda_{2}\right)}$ & $(1,2)$ & $X_{1}<X_{2}$ & $I_{121}$ \\
\hline$U_{2}<U_{0}<U_{1}$ & $\frac{\lambda_{0} \lambda_{2}}{\left(\lambda_{0}+\lambda_{1}\right)\left(\lambda_{0}+\lambda_{1}+\lambda_{2}\right)}$ & $(0,2)$ & $X_{1}>X_{2}$ & $I_{02}$ \\
\hline$U_{2}<U_{1}<U_{0}$ & $\frac{\lambda_{1} \lambda_{2}}{\left(\lambda_{0}+\lambda_{1}\right)\left(\lambda_{0}+\lambda_{1}+\lambda_{2}\right)}$ & $(1,2)$ & $X_{1}>X_{2}$ & $I_{122}$ \\
\hline
\end{tabular}

We use the following notation;

$$
\begin{gathered}
\gamma=\left(\lambda_{0}, \lambda_{1}, \lambda_{2}, \alpha\right), \quad a_{0}=E\left(U_{0} \mid U_{0}<\min \left\{U_{1}, U_{2}\right\}\right)=\frac{1}{\left(\lambda_{0}+\lambda_{1}+\lambda_{2}\right)^{1 / \alpha}} \Gamma\left(\frac{1}{\alpha}+1\right), \\
u_{1}=\frac{\lambda_{0}}{\lambda_{0}+\lambda_{2}}=P\left(\Delta_{2}=0 \mid X_{1}<X_{2}\right), \quad u_{2}=\frac{\lambda_{2}}{\lambda_{0}+\lambda_{2}}=P\left(\Delta_{2}=2 \mid X_{1}<X_{2}\right), \\
v_{1}=\frac{\lambda_{0}}{\lambda_{0}+\lambda_{1}}=P\left(\Delta_{1}=0 \mid X_{1}>X_{2}\right), \quad v_{2}=\frac{\lambda_{1}}{\lambda_{0}+\lambda_{1}}=P\left(\Delta_{1}=1 \mid X_{1}>X_{2}\right),
\end{gathered}
$$

and $n_{0}=\left|I_{0}\right|=m-n_{1}-n_{2}$ and $m \geq n$ is a random number which has negative binomial distribution with parameters $\frac{\lambda_{1}+\lambda_{2}}{\lambda_{0}+\lambda_{1}+\lambda_{2}}$ and $n_{1}+n_{2}$. We further use,

$$
\tilde{n}_{0}=E\left(n_{0} \mid n_{1}, n_{2}\right)=\left(n_{1}+n_{2}\right) \frac{\lambda_{0}}{\lambda_{1}+\lambda_{2}} .
$$

Therefore, based on the observations $\left\{\left(y_{1 i}, y_{2 i}\right), i=1, \ldots, n\right\}$, we write the pseudo loglikelihood function by replacing the missing observation by its expected value. It becomes

$$
\begin{aligned}
l_{\text {pseudo }}(\gamma)= & \tilde{n}_{0}\left(\ln \alpha+\ln \lambda_{0}\right)+\widetilde{n}_{0}(\alpha-1) \ln a_{0}-\widetilde{n}_{0}\left(\lambda_{0}+\lambda_{1}+\lambda_{2}\right) a_{0}^{\alpha} \\
& +u_{1}\left(n_{1}\left(\ln \alpha+\ln \lambda_{1}\right)-\lambda_{1} \sum_{i \in I_{1}} y_{1 i}^{\alpha}+(\alpha-1) \sum_{i \in I_{1}} \ln y_{1 i}+n_{1}\left(\ln \alpha+\ln \lambda_{0}\right)\right.
\end{aligned}
$$




$$
\begin{aligned}
& \left.-\left(\lambda_{0}+\lambda_{2}\right) \sum_{i \in I_{1}} y_{2 i}^{\alpha}+(\alpha-1) \sum_{i \in I_{1}} \ln y_{2 i}\right) \\
& +u_{2}\left(n_{1}\left(\ln \alpha+\ln \lambda_{1}\right)-\lambda_{1} \sum_{i \in I_{1}} y_{1 i}^{\alpha}+(\alpha-1) \sum_{i \in I_{1}} \ln y_{1 i}+n_{1}\left(\ln \alpha+\ln \lambda_{2}\right)\right. \\
& \left.-\left(\lambda_{0}+\lambda_{2}\right) \sum_{i \in I_{1}} y_{2 i}^{\alpha}+(\alpha-1) \sum_{i \in I_{1}} \ln y_{2 i}\right) \\
& +v_{1}\left(n_{2}\left(\ln \alpha+\ln \lambda_{2}\right)-\lambda_{2} \sum_{i \in I_{2}} y_{2 i}^{\alpha}+(\alpha-1) \sum_{i \in I_{2}} \ln y_{2 i}+n_{2}\left(\ln \alpha+\ln \lambda_{0}\right)\right. \\
& \left.-\left(\lambda_{0}+\lambda_{1}\right) \sum_{i \in I_{2}} y_{1 i}^{\alpha}+(\alpha-1) \sum_{i \in I_{2}} \ln y_{1 i}\right) \\
& +v_{2}\left(n_{2}\left(\ln \alpha+\ln \lambda_{2}\right)-\lambda_{2} \sum_{i \in I_{2}} y_{2 i}^{\alpha}+(\alpha-1) \sum_{i \in I_{2}} \ln y_{2 i}+n_{2}\left(\ln \alpha+\ln \lambda_{1}\right)\right. \\
& \left.-\left(\lambda_{0}+\lambda_{1}\right) \sum_{i \in I_{2}} y_{1 i}^{\alpha}+(\alpha-1) \sum_{i \in I_{2}} \ln y_{1 i}\right) \\
& =\left(\widetilde{n}_{0}+2 n_{1}+2 n_{2}\right) \ln \alpha+(\alpha-1)\left[\tilde{n}_{0} \ln a_{0}+\sum_{i \in I_{1} \cup I_{2}}\left(\ln y_{1 i}+\ln y_{2 i}\right)\right]- \\
& \lambda_{0}\left[\widetilde{n}_{0} a_{0}^{\alpha}+\sum_{i \in I_{1}} y_{2 i}^{\alpha}+\sum_{i \in I_{2}} y_{1 i}^{\alpha}\right]+\left(\widetilde{n}_{0}+u_{1} n_{1}+v_{1} n_{2}\right) \ln \lambda_{0}- \\
& \lambda_{1}\left[\widetilde{n}_{0} a_{0}^{\alpha}+\sum_{i \in I_{1} \cup I_{2}} y_{1 i}^{\alpha}\right]+\ln \lambda_{1}\left(n_{1}+v_{2} n_{2}\right)- \\
& \lambda_{2}\left[\widetilde{n}_{0} a_{0}^{\alpha}+\sum_{i \in I_{1} \cup I_{2}} y_{2 i}^{\alpha}\right]+\ln \lambda_{2}\left(u_{2} n_{1}+n_{2}\right) .
\end{aligned}
$$

Therefore, for fixed $\alpha$, at each step, the pseudo maximum likelihood estimates of $\lambda_{0}, \lambda_{1}$ and $\lambda_{2}$ can be obtained as

$$
\widehat{\lambda}_{0}(\alpha)=\frac{\widetilde{n}_{0}+u_{1} n_{1}+v_{1} n_{2}}{\widetilde{n}_{0} a_{0}^{\alpha}+\sum_{i \in I_{2}} y_{1 i}^{\alpha}+\sum_{i \in I_{1}} y_{2 i}^{\alpha}}, \quad \widehat{\lambda}_{1}(\alpha)=\frac{n_{1}+v_{2} n_{2}}{\widetilde{n}_{0} a_{0}^{\alpha}+\sum_{i \in I} y_{1 i}^{\alpha}}, \quad \widehat{\lambda}_{2}(\alpha)=\frac{n_{2}+u_{2} n_{1}}{\widetilde{n}_{0} a_{0}^{\alpha}+\sum_{i \in I} y_{2 i}^{\alpha}} .
$$

The pseudo maximum likelihood estimate of $\alpha$ at each step can be obtained by maximizing the pseudo profile log-likelihood function $l_{\text {pseudo }}\left(\alpha, \widehat{\lambda}_{0}(\alpha), \widehat{\lambda}_{1}(\alpha), \widehat{\lambda}_{2}(\alpha)\right)$. It has been shown 
in the Appendix A (see Lemma 1) that the pseudo log-likelihood function is a unimodal function and therefore it has the unique maximum.

The maximization of $l_{\text {pseudo }}\left(\alpha, \widehat{\lambda}_{0}(\alpha), \widehat{\lambda}_{1}(\alpha), \widehat{\lambda}_{2}(\alpha)\right)$ with respect to $\alpha$ can be performed by solving the following fixed point equation

$$
g(\alpha)=\alpha
$$

where $g(\alpha)=\left(\widetilde{n}_{0}+2 n_{1}+2 n_{2}\right)[h(\alpha)]^{-1}$, and

$$
\begin{aligned}
h(\alpha)= & \hat{\lambda}_{0}(\alpha)\left[\widetilde{n}_{0} a_{0}^{\alpha} \ln a_{0}+\sum_{i \in I_{1}} y_{2 i}^{\alpha} \ln y_{2 i}+\sum_{i \in I_{2}} y_{1 i}^{\alpha} \ln y_{2 i}\right]+\widehat{\lambda}_{1}(\alpha)\left[\widetilde{n}_{0} a_{0}^{\alpha} \ln a_{0}+\sum_{i \in I} y_{1 i}^{\alpha} \ln y_{1 i}\right]+ \\
& \hat{\lambda}_{2}(\alpha)\left[\widetilde{n}_{0} a_{0}^{\alpha} \ln a_{0}+\sum_{i \in I} y_{2 i}^{\alpha} \ln y_{2 i}\right]-\left[\left[\widetilde{n}_{0} \ln a_{0}+\sum_{i \in I} \ln y_{1 i}+\sum_{i \in I} \ln y_{2 i}\right] .\right.
\end{aligned}
$$

Note that solving (21) is quite simple. We suggest to use a similar algorithm as of Kundu and Gupta [8]. Start with an initial guess $\alpha^{(0)}$, then $\alpha^{(1)}=g\left(\alpha^{(0)}\right)$, similarly, $\alpha^{(2)}=g\left(\alpha^{(1)}\right)$ and so on. Continue the process until the convergence is obtained. Alternatively, the maximization of the pseudo log-likelihood function can be performed by using Newton-Raphson or by bisection method also, but it is observed that they take larger number of iterations than the proposed one.

Now we can provide the EM algorithm as follows. Start with an initial guess of $\alpha, \lambda_{0}, \lambda_{1}$ and $\lambda_{2}$ as $\alpha^{(0)}, \lambda_{0}^{(0)}, \lambda_{1}^{(0)}, \lambda_{2}^{(0)}$ respectively.

- Obtain $u_{1}, u_{2}, v_{1}, v_{2}, \widetilde{n}_{0}$ and $a_{0}$ from $\alpha^{(0)}, \lambda_{0}^{(0)}, \lambda_{1}^{(0)}, \lambda_{2}^{(0)}$.

- Maximize $l_{\text {pseudo }}\left(\alpha, \widehat{\lambda}_{0}(\alpha), \widehat{\lambda}_{1}(\alpha), \widehat{\lambda}_{2}(\alpha)\right)$ and obtain $\alpha^{(1)}$.

- Obtain $\lambda_{0}^{(1)}=\widehat{\lambda}_{0}\left(\alpha^{(1)}\right), \lambda_{1}^{(1)}=\widehat{\lambda}_{1}\left(\alpha^{(1)}\right), \lambda_{2}^{(1)}=\widehat{\lambda}_{2}\left(\alpha^{(1)}\right)$, using (20).

- Update $u_{1}, u_{2}, v_{1}, v_{2}, \widetilde{n}_{0}$ and $a_{0}$ from $\alpha^{(1)}, \lambda_{0}^{(1)}, \lambda_{1}^{(1)}, \lambda_{2}^{(1)}$.

- Continue the process until the convergence is met. 
Comment: Note that the above EM algorithm can be easily used for the BBBE model. In that case since $\alpha=1$, therefore Step 2 is not needed. From the $i-t h$ step $(i+1)-t h$ step can be easily obtained from (20) using $\alpha=1$.

\section{Data Analysis}

In this section we analyze one data set for illustrative purposes. This data set has been obtained from Johnson and Wichern [6] (page 374). It represents the bone mineral density (BMD) measured in $\mathrm{gm} / \mathrm{cm}^{2}$ for 24 children after one year of birth. This bivariate data represent the BMD for Dominant Ulna and Ulna bones and they are as follows;

Data Set: (0.869 0.964), (0.602 0.689), (0.765 0.738), (0.761 0.698), (0.551 0.619), (0.753 0.515), (0.708 0.787), (0.687 0.715), (0.844 0.656), (0.869 0.789), (0.654 0.726), (0.692 0.526), (0.670 0.580), (0.823 0.773), (0.746 0.729), (0.656 0.506), (0.693 0.740), (0.883 0.785), (0.577 0.627), (0.802 0.769), (0.540 0.498), (0.804 0.779), (0.570 0.634), (0.585 0.640).

Just to get an idea about the hazard functions of the marginals we provide the scaled TTT plot as suggested by Aarset [1], which provides an idea of the shape of the hazard function of a distribution. For a family with survival function $S(y)=1-F(y)$, the scaled TTT transform with $H^{-1}(u)=\int_{0}^{F^{-1}(u)} S(y) d y$ defined for $0<u<1$ is $g(u)=H^{-1}(u) / H^{-1}(1)$. The corresponding empirical version of the scaled TTT transform is given by $g_{n}(r / n)=$ $H_{n}^{-1}(r / n) / H_{n}^{-1}(1)=\left[\sum_{i=1}^{r} y_{i: n}+(n-r) y_{r: n}\right]$, where $r=1, \ldots, n$ and $y_{i: n}, i=1, \ldots, n$ represent the order statistics of the sample. It has been shown by Aarset [1] that the scaled TTT transform is convex (concave) if the hazard rate is decreasing (increasing) and for bathtub (unimodal) shaped hazard rate, the scaled TTT transform is first convex (concave) and then concave (convex). In this case the scale TTT transform of BMDs of Dominant radius and Radius bones are given in Figure 3. It clearly indicates that both the marginals have in- 


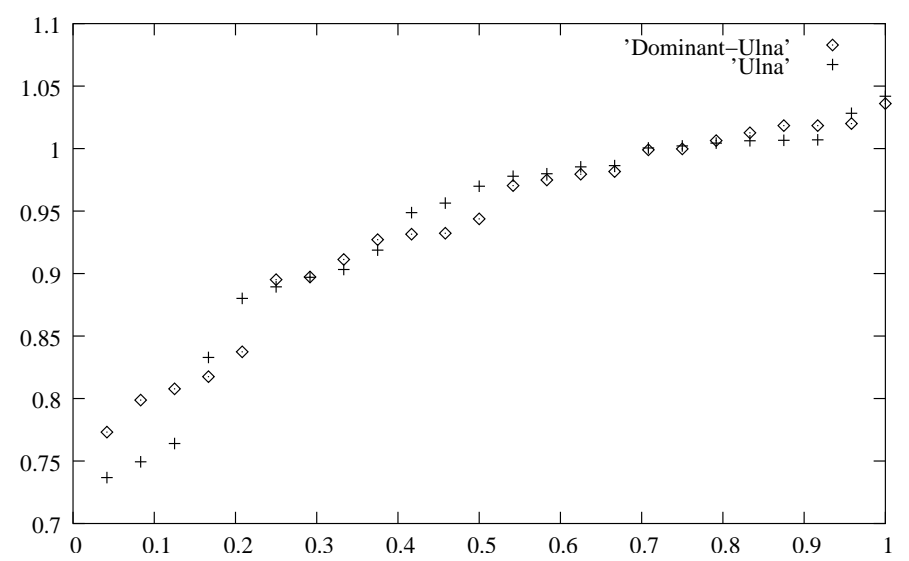

Figure 3: The scaled TTT transform of the BMDs of Dominant Ulna and Ulna bones.

creasing hazard rates. The correlation coefficient between the two variables is 0.628 and that shows they are positively correlated. We have fitted the Weibull distribution to the minimum of the two variables. The MLEs of the shape and scale parameters are 7.0795 and 11.8922 respectively. The Kolmogorov-Smirnov (K-S) distance between the empirical distribution function and the fitted distribution function is 0.141 and the corresponding $p$-value is 0.731 . It implies that the Weibull distribution fits very well to the minimum of the two variables. Therefore it is not unreasonable to fit the BBBW model in this case.

We have used the proposed EM algorithm to compute the MLEs of the unknown parameters. We start the EM algorithm with the initial estimate of $\alpha$ as 7.00. We do not have immediate initial estimates of $\lambda_{0}, \lambda_{1}$ and $\lambda_{2}$, but we have an initial estimate of $\lambda_{0}+\lambda_{1}+\lambda_{2}$, i.e. 11.892. So we start the iterative process with the initial estimates of $\lambda_{0}, \lambda_{1}$ and $\lambda_{2}$ as 3.00 each. The EM algorithm provides the estimates of $\alpha, \lambda_{0}, \lambda_{1}$ and $\lambda_{2}$ as $7.2547,8.0262$, 3.6399, 5.1833 respectively. Using the method proposed by Louis [10], the 95\% confidence intervals of $\alpha, \lambda_{0}, \lambda_{1}$ and $\lambda_{2}$ become $(6.3381,8.1712),(5.9827,10.0698),(2.0628,5.2170)$ and (3.2047, 7.1619) respectively.

Now we would like to see how good is the fit. We do not have any proper bivariate 
goodness of fit test like the univariate case. We examine the marginals and definitely it provide some indications about the goodness of fit. The empirical survival functions and the corresponding fitted survival functions for Dominant-Ulma and Ulma are reported in Figure 4. The K-S distances between empirical distribution functions and the corresponding
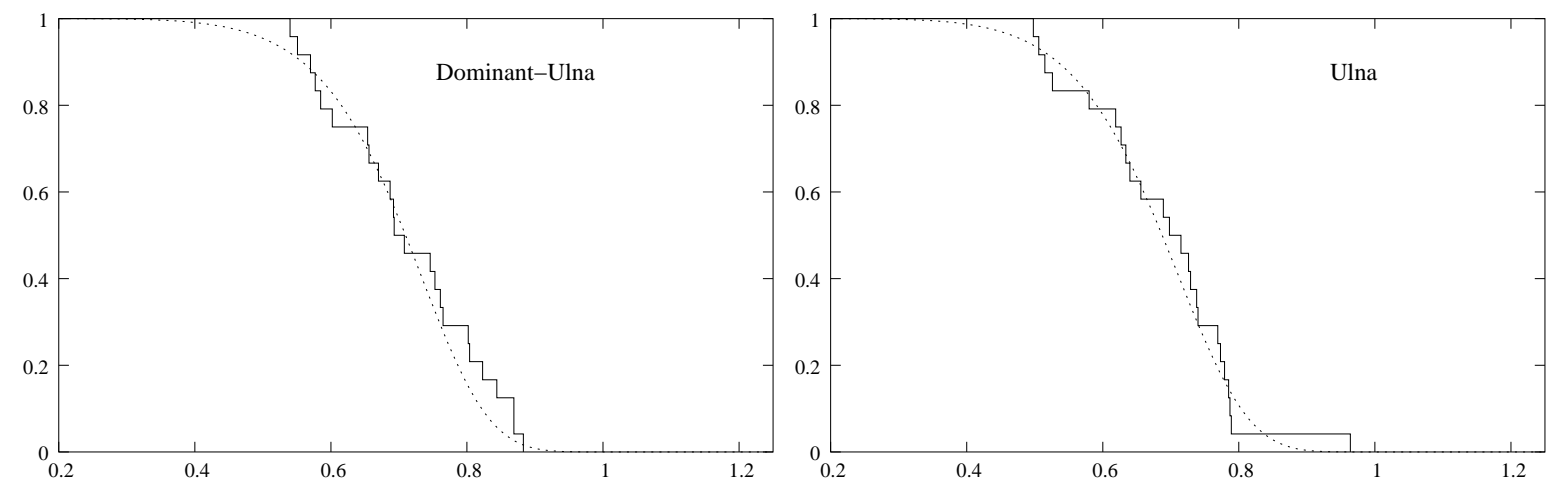

Figure 4: The empirical survival function and fitted survival function for Dominant-Ulna and Ulna.

fitted distribution functions of the marginals are 0.141 and 0.109 and the corresponding $p$ values are 0.730 and 0.936 respectively. Moreover, it has also been observed that the Weibull distribution fits the minimum also quite well. Considering all these points, we can say that BBBW may be used in this case for analyzing the data.

\section{Conclusions}

In this paper we have introduced a new absolutely continuous bivariate model following the approach of Block and Basu [3]. Block and Basu [3] obtained the BBBE model from the Marshall-Olkin bivariate exponential model by removing the singular component. Exactly in a similar manner we have obtained the BBBW model from the Marshall-Olkin bivariate Weibull model by removing the singular component. It has an absolutely continuous probability density function and we have studied several properties of this new distribution. This 
model has four unknown parameters and we have suggested an EM algorithm to compute the MLEs. It is observed that the proposed model work quite well for data analysis purposes.

Now we discuss some of the open problems. Note that although we have defined the model for the bivariate case but it is possible to define even for the multivariate case. It will be interesting to see how the estimation procedure can be generalized in this case. Moreover, Bayesian analysis of the proposed should be possible along the same line as Pena and Gupta [12]. More work is needed in these directions.

\section{ACKNOWLEDGEMENTS}

The authors would like to thank the referee and the associate editor for carefully reading the manuscript and made some valuable comments which had greatly improved the earlier draft of the manuscript.

\section{APPENDIX A:}

LEmma 1: The pseudo log-likelihood function $l_{\text {pseudo }}\left(\alpha, \widehat{\lambda}_{0}(\alpha), \widehat{\lambda}_{1}(\alpha), \widehat{\lambda}_{2}(\alpha)\right)=l_{\text {pseudo }}(\alpha)$ (say), is unimodal.

Proof: The pseudo log-likelihood function without the additive constant can be written as

$$
\begin{aligned}
l_{\text {pseudo }}(\alpha)= & \left(\widetilde{n}_{0}+2 n\right) \ln \alpha+(\alpha-1)\left[\widetilde{n}_{0} \ln a_{0}+\sum_{i \in I} \ln y_{1 i}+\sum_{i \in I} \ln y_{2 i}\right]- \\
& \left(u_{2} n_{1}+v_{1} n_{2}\right) \ln \left(\widetilde{n}_{0} a_{0}^{\alpha}+\sum_{i \in I_{2}} y_{1 i}^{\alpha}+\sum_{i \in I_{1}} y_{2 i}^{\alpha}\right) \\
& -\left(n_{1}+v_{1} n_{2}\right) \ln \left(\widetilde{n}_{0} a_{0}^{\alpha}+\sum_{i \in I} y_{1 i}^{\alpha}\right)-\left(n_{2}+u_{2} n_{1}\right) \ln \left(\widetilde{n}_{0} a_{0}^{\alpha}+\sum_{i \in I} y_{2 i}^{\alpha}\right) .
\end{aligned}
$$

First we prove that if $g(\alpha)=\sum_{i \in I} y_{1 i}^{\alpha}$, then $\ln g(\alpha)$ is concave. From

$$
g^{\prime}(\alpha)=\sum_{i \in I} y_{1 i}^{\alpha} \ln y_{1 i}, \quad g^{\prime \prime}(\alpha)=\sum_{i \in I} y_{1 i}^{\alpha}\left(\ln y_{1 i}\right)^{2}
$$


and

$$
\left(\sum_{i \in I} y_{1 i}^{\alpha}\left(\ln y_{1 i}\right)^{2}\right) \times\left(\sum_{i \in I} y_{1 i}^{\alpha}\right)-\left(\sum_{i \in I} y_{1 i}^{\alpha}\left(\ln y_{1 i}\right)\right)^{2}=\sum_{i<j} y_{1 i}^{\alpha} y_{1 j}^{\alpha}\left(y_{1 i}-y_{1 j}\right)^{2} \geq 0
$$

it implies

$$
g^{\prime \prime}(\alpha) g(\alpha) \geq\left(g^{\prime}(\alpha)\right)^{2}
$$

Therefore, $\ln g(\alpha)$ is concave. Similarly, it follows that

$$
\ln \left(\widetilde{n}_{0} a_{0}^{\alpha}+\sum_{i \in I_{2}} y_{1 i}^{\alpha}+\sum_{i \in I_{1}} y_{2 i}^{\alpha}\right), \ln \left(\widetilde{n}_{0} a_{0}^{\alpha}+\sum_{i \in I} y_{i i}^{\alpha}\right) \ln \left(\widetilde{n}_{0} a_{0}^{\alpha}+\sum_{i \in I} y_{2 i}^{\alpha}\right)
$$

are also concave functions. It implies $l_{\text {pseudo }}(\alpha)$ is a concave function. Now unimodality follows by observing that as $\alpha$ tends to 0 or $\infty, l_{\text {pseudo }}(\alpha)$ tends to $-\infty$.

\section{Appendix B:}

\section{ExpeCted Fisher Information MATRix}

Let the Fisher information matrix be;

$$
I=-E\left[\begin{array}{cccc}
\frac{\partial^{2} l}{\partial \alpha^{2}} & \frac{\partial^{2} l}{\partial \alpha \partial \lambda_{0}} & \frac{\partial^{2} l}{\partial \alpha \partial \lambda_{1}} & \frac{\partial^{2} l}{\partial \alpha \partial \lambda_{2}} \\
\frac{\partial^{2} l}{\partial \lambda_{0} \partial \alpha} & \frac{\partial^{2} l}{\partial \lambda_{0}^{2}} & \frac{\partial^{2} l}{\partial \lambda_{0} \partial \lambda_{1}} & \frac{\partial^{2} l}{\partial \lambda_{0} \partial \lambda_{2}} \\
\frac{\partial^{2} l}{\partial \lambda_{1} \partial \alpha} & \frac{\partial^{2} l}{\partial \lambda_{1} \partial \lambda_{0}} & \frac{\partial^{2} l}{\partial \lambda_{1}^{2}} & \frac{\partial^{2} l}{\partial \lambda_{1} \partial \lambda_{2}} \\
\frac{\partial^{2} l}{\partial \lambda_{2} \partial \alpha} & \frac{\partial^{2} l}{\partial \lambda_{2} \partial \lambda_{0}} & \frac{\partial^{2} l}{\partial \lambda_{2} \partial \lambda_{1}} & \frac{\partial^{2} l}{\partial \lambda_{2}^{2}}
\end{array}\right]=\left[\begin{array}{cccc}
a_{11} & a_{12} & a_{13} & a_{14} \\
a_{21} & a_{22} & a_{23} & a_{24} \\
a_{31} & a_{32} & a_{33} & a_{34} \\
a_{41} & a_{42} & a_{43} & a_{44}
\end{array}\right]
$$

Before, providing all the elements explicitly we introduce the following notations. If $Z \sim$ $\mathrm{WE}(\alpha, \lambda)$, then

$$
\begin{aligned}
E\left(Z^{\alpha} \ln Z^{\alpha}\right) & \left.=\frac{1}{\lambda}(\psi(2)-\ln (\lambda))=\xi(\lambda) \quad \text { Let }\right) \\
E\left(Z^{\alpha}\left(\ln Z^{\alpha}\right)^{2}\right) & =\frac{1}{\lambda}\left(\psi^{\prime}(2)+(\psi(2)-\ln (\lambda))^{2}\right)=\eta(\lambda),
\end{aligned}
$$

here $\psi(\cdot)$ and $\psi^{\prime}(\cdot)$ are the digamma and polygamma functions. We also need the following results:

$$
E\left(n_{1}\right)=\frac{n \lambda_{1}}{\lambda_{1}+\lambda_{2}}, \quad E\left(n_{2}\right)=\frac{n \lambda_{2}}{\lambda_{1}+\lambda_{2}}
$$


Then

$$
\begin{aligned}
& a_{11}=\frac{n}{\alpha^{2}}\left[2+c \lambda_{1} \eta\left(\lambda_{0}+\lambda_{1}\right)+c \lambda_{2} \eta\left(\lambda_{0}+\lambda_{2}\right)\right], \\
& a_{22}=n \times\left[\frac{1}{\left(\lambda_{0}+\lambda_{1}+\lambda_{2}\right)^{2}}+\frac{\lambda_{1}}{\left(\lambda_{1}+\lambda_{2}\right)\left(\lambda_{0}+\lambda_{2}\right)^{2}}+\frac{\lambda_{2}}{\left(\lambda_{1}+\lambda_{2}\right)\left(\lambda_{0}+\lambda_{1}\right)^{2}}\right], \\
& a_{33}=n \times\left[\frac{1}{\left(\lambda_{0}+\lambda_{1}+\lambda_{2}\right)^{2}}-\frac{\lambda_{1}}{\left(\lambda_{1}+\lambda_{2}\right)^{3}}+\frac{1}{\lambda_{1}\left(\lambda_{1}+\lambda_{2}\right)}+\frac{\lambda_{2}}{\left(\lambda_{1}+\lambda_{2}\right)\left(\lambda_{0}+\lambda_{1}\right)^{2}}\right] \text {, } \\
& a_{44}=n \times\left[\frac{1}{\left(\lambda_{0}+\lambda_{1}+\lambda_{2}\right)^{2}}-\frac{\lambda_{2}}{\left(\lambda_{1}+\lambda_{2}\right)^{3}}+\frac{\lambda_{1}}{\left(\lambda_{1}+\lambda_{2}\right)\left(\lambda_{0}+\lambda_{2}\right)^{2}}+\frac{1}{\lambda_{2}\left(\lambda_{1}+\lambda_{2}\right)}\right] \text {, } \\
& a_{12}=a_{21}=\frac{n}{\alpha} \xi\left(\lambda_{0}+\lambda_{1}+\lambda_{2}\right) \text {, } \\
& a_{13}=a_{31}=\frac{c n}{\alpha}\left[\xi\left(\lambda_{0}+\lambda_{1}\right)-\frac{\lambda_{0}}{\lambda_{0}+\lambda_{1}+\lambda_{2}} \xi\left(\lambda_{0}+\lambda_{1}+\lambda_{2}\right)\right], \\
& a_{14}=a_{41}=\frac{c n}{\alpha}\left[\xi\left(\lambda_{0}+\lambda_{2}\right)-\frac{\lambda_{0}}{\lambda_{0}+\lambda_{1}+\lambda_{2}} \xi\left(\lambda_{0}+\lambda_{1}+\lambda_{2}\right)\right] \text {, } \\
& a_{23}=a_{32}=n \times\left[\frac{1}{\left(\lambda_{0}+\lambda_{1}+\lambda_{2}\right)^{2}}+\frac{\lambda_{2}}{\left(\lambda_{1}+\lambda_{2}\right)\left(\lambda_{0}+\lambda_{1}\right)^{2}}\right] \text {, } \\
& a_{24}=a_{42}=n \times\left[\frac{1}{\left(\lambda_{0}+\lambda_{1}+\lambda_{2}\right)^{2}}+\frac{\lambda_{1}}{\left(\lambda_{1}+\lambda_{2}\right)\left(\lambda_{0}+\lambda_{2}\right)^{2}}\right] \text {, } \\
& a_{34}=a_{43}=\frac{n}{\left(\lambda_{1}+\lambda_{2}\right)^{2}}-\frac{n}{\left(\lambda_{0}+\lambda_{1}+\lambda_{2}\right)^{2}} \text {. }
\end{aligned}
$$

\section{OBSERVED Fisher Information MATRiX}

To compute the observed information matrix, we use the same notation as of Louis [10]. If the matrix $\mathbf{S}=\left(\left(S_{i j}\right)\right)$ denotes the Hessian matrix and the vector $\mathbf{U}=\left(\left(U_{i}\right)\right)$ denotes the gradient vector of the pseudo log-likelihood function, then the observed Fisher information matrix can be obtained as $\mathbf{S}-\mathbf{U U}^{T}$. Below we provide the elements of the matrix $\mathbf{S}$ and the vector $\mathbf{U}$.

$$
\begin{aligned}
S_{11}= & \frac{\widetilde{n}_{0}+2 n_{1}+2 n_{2}}{\widehat{\alpha}^{2}}+\widehat{\lambda}_{0}\left[\sum_{i \in I_{2}} y_{1 i}^{\widehat{\alpha}}\left(\ln y_{1 i}\right)^{2}+\sum_{i \in I_{1}} y_{2 i}^{\widehat{\alpha}}\left(\ln y_{2 i}\right)^{2}+\widetilde{n}_{0} a_{0}^{\widehat{\alpha}}\left(\ln a_{0}\right)^{2}\right], \\
& +\widehat{\lambda}_{1}\left[\sum_{i \in I_{1} \cup I_{2}} y_{1 i}^{\widehat{\alpha}}\left(\ln y_{1 i}\right)^{2}+\widetilde{n}_{0} a_{0}^{\widehat{\alpha}}\left(\ln a_{0}\right)^{2}\right]+\widehat{\lambda}_{2}\left[\sum_{i \in I_{1} \cup I_{2}} y_{2 i}^{\widehat{\alpha}}\left(\ln y_{2 i}\right)^{2}+\widetilde{n}_{0} a_{0}^{\widehat{\alpha}}\left(\ln a_{0}\right)^{2}\right], \\
S_{12}= & S_{21}=\sum_{i \in I_{2}} y_{1 i}^{\widehat{\alpha}} \ln y_{1 i}+\sum_{i \in I_{1}} y_{2 i}^{\widehat{\alpha}} \ln y_{2 i}+\widetilde{n}_{0} a_{0}^{\widehat{\alpha}} \ln a_{0},
\end{aligned}
$$




$$
\begin{aligned}
& S_{13}=S_{31}=\sum_{i \in I_{1} \cup I_{2}} y_{1 i}^{\widehat{\alpha}} \ln y_{1 i}+\widetilde{n}_{0} a_{0}^{\widehat{\alpha}} \ln a_{0}, \\
& S_{14}=S_{41}=\sum_{i \in I_{1} \cup I_{2}} y_{2 i}^{\widehat{\alpha}} \ln y_{2 i}+\widetilde{n}_{0} a_{0}^{\widehat{\alpha}} \ln a_{0}, \\
& S_{22}=\frac{\tilde{n}_{0}+u_{1} n_{1}+v_{1} n_{2}}{\widehat{\lambda}_{0}^{2}}, \quad S_{23}=S_{32}=0, \\
& S_{33}=\frac{n_{1}+v_{2} n_{2}}{\widehat{\lambda}_{1}^{2}}, \quad S_{34}=S_{43}=0, \quad S_{44}=\frac{u_{2} n_{1}+n_{2}}{\widehat{\lambda}_{2}^{2}} . \\
& U_{1}=\frac{\widetilde{n}_{0}+2 n_{1}+2 n_{2}}{\widehat{\alpha}}+\left[\sum_{i \in I_{1} \cup I_{2}} \ln y_{1 i}+\sum_{i \in I_{1} \cup I_{2}} \ln y_{2 i}+\widetilde{n}_{0} \ln a_{0}\right]- \\
& \widehat{\lambda}_{1}\left[\sum_{i \in I_{1} \cup I_{2}} y_{1 i}^{\widehat{\alpha}} \ln y_{1 i}+\widetilde{n}_{0} a_{0}^{\widehat{\alpha}} \ln a_{0}\right]-\widehat{\lambda}_{2}\left[\sum_{i \in I_{1} \cup I_{2}} y_{2 i}^{\widehat{\alpha}} \ln y_{2 i}+\widetilde{n}_{0} a_{0}^{\widehat{\alpha}} \ln a_{0}\right] \text {, } \\
& -\widehat{\lambda}_{0}\left[\sum_{i \in I_{2}} y_{1 i}^{\widehat{\alpha}} \ln y_{1 i}+\sum_{i \in I_{1}} y_{2 i}^{\widehat{\alpha}} \ln y_{2 i}+\widetilde{n}_{0} a_{0}^{\widehat{\alpha}} \ln a_{0}\right] \text {, } \\
& U_{2}=\frac{\widetilde{n}_{0}+u_{1} n_{1}+v_{1} n_{2}}{\widehat{\lambda}_{0}}-\left[\sum_{i \in I_{2}} y_{1 i}^{\widehat{\alpha}}+\sum_{i \in I_{1}} y_{2 i}^{\widehat{\alpha}}+\widetilde{n}_{0} a_{0}^{\widehat{\alpha}}\right] \text {, } \\
& U_{3}=\frac{n_{1}+v_{2} n_{2}}{\widehat{\lambda}_{1}}-\left[\sum_{i \in I_{1} \cup I_{2}} y_{1 i}^{\widehat{\alpha}}+\widetilde{n}_{0} a_{0}^{\widehat{\alpha}}\right] \text {, } \\
& U_{4}=\frac{u_{2} n_{1}+n_{2}}{\widehat{\lambda}_{2}}-\left[\sum_{i \in I_{1} \cup I_{2}} y_{2 i}^{\widehat{\alpha}}+\tilde{n}_{0} a_{0}^{\widehat{\alpha}}\right] \text {. }
\end{aligned}
$$

\section{References}

[1] Aarset, M.V. (1987), "How to identify a bathtub shaped hazard rate?", IEEE Transactions on Reliability, vol. 36, 106 - 108.

[2] Basu, A.P. (1971), "Bivariate hazard rate", Journal of the American Statistical Association, vol. 66, 103 - 104.

[3] Block, H., Basu, A. P. (1974), "A continuous bivariate exponential extension", Journal of the American Statistical Association, vol. 69, 1031 - 1037. 
[4] Harris, R. (1970), "A multivariate defintion for increasing hazard rate distribution functions", The Annals of Mathematical Statistics, vol. 41, 713 - 717.

[5] Johnson, N.L. and Kotz, S. (1975), "A vector multivariate hazard rate", Journal of Multivariate Analysis, vol. 5, 53-66.

[6] Johnson, R.A. and Wichern, D.W. (1999), Applied Multivariate Analysis, Fourth Edition, Prentice Hall, New Jersey.

[7] Karlis, D. (2003), "ML estimation for multivarite shock models via an EM algorithm", Annals of the Institute of Statistical Mathematics, 55, 817-830.

[8] Kundu, D. and Gupta, R.D. (2006), "Estimation of $\mathrm{P}(\mathrm{Y}<\mathrm{X})$ for Weibull distribution", IEEE Transactions on Reliability, vol. 55, 270 - 280.

[9] Lehmann, E.L. (1966), "Some concepts of dependence", The Annals of Mathematical Statistics, vol. 37, 1137 - 1153.

[10] Louis, T.A. (1982), "Finding the observed information matrix when using the EM algorithm", Journal of the Royal Statistical Society, Ser B, vol. 44, 226 - 233.

[11] Marshall, A.W. and Olkin, I. (1967), "A multivariate exponential distribution", Journal of the American Statistical Association, vol. 62, 30 - 44.

[12] Peña, E. A. and Gupta, A. K. (1990), "Bayes estimation for the Marshall-Olkin exponential distribution", Journal of the Royal Statistical Society. Ser. B vol. 52, 379-389. 